\title{
Camptodactyly: An unsolved area of plastic surgery
}

\author{
Veena Singh ${ }^{1}$, Ansarul Haq ${ }^{1}$, Puja Priyadarshini ${ }^{1}$, Purshottam Kumar ${ }^{2}$ \\ Departments of ${ }^{1}$ Burns \& Plastic Surgery and ${ }^{2}$ General Surgery, All India Institute of Medical Sciences Patna, Patna, India
}

Background Camptodactyly refers to permanent flexion contracture at the proximal interphalangeal joint. Most cases are limited to fifth-finger involvement. Although common, the treatment of camptodactyly is controversial. Many published studies have emphasized conservative treatment, while others have described surgical procedures. The problem with this deformity is that it presents in several forms, which means that there is no single model for effective treatment. The aim of this paper is to present the difficulties encountered with this condition and the management thereof on an individual basis.

Methods This is a case series of 14 patients (nine males, five females) who underwent surgical treatment. The results were classified using the method from Mayo Clinic as excellent, good, fair, and poor.

Results Fourteen patients with 15 fingers underwent surgery, and the results achieved were as follows: excellent, 0 ; good, 1 ; fair, 6 ; poor, 8 . The treatment of camptodactyly still remains controversial, and hence proper planning individualized to each patient is needed to achieve the maximal improvement with realistic goals.

Conclusions Although we performed individualised surgery, our careful follow-up was not able to identify any method as superior over another with respect to gain in extension and loss of flexion. We therefore propose that the extensor mechanism should not be disturbed during surgery to treat camptodactyly cases.

Keywords Fingers / Joints / Congenital abnormalities / Ankylosis
Correspondence: Veena Singh Department of Burns \& Plastic Surgery, All India Institute of Medical Sciences Patna, Patna 801507, India

Tel: +91-9905755111

Fax: +91-612-2451109

E-mail: drsveena@gmail.com

Received: 14 Apr 2017 • Revised: 17 Apr 2018 • Accepted: 22 Jun 2018

pISSN: 2234-6163 • elSSN: 2234-6171 • https://doi.org/10.5999/aps.2017.00759 • Arch Plast Surg 2018;45:363-366

The article was presented in the Best Paper Session at the 40th Annual Conference of the Indian Society for Surgery of the Hand 2016, September 15-17, 2016, at Ranchi, India.

\section{INTRODUCTION}

Camptodactyly is a relatively rare hand anomaly, and this condition involves varying degrees of congenital or acquired flexion contracture of the fingers at the proximal interphalangeal (PIP) joint, unilaterally or bilaterally $[1,2]$. The fifth finger is mostly involved, and the entity can occur singly or have a syndromic association. Several aetiologies have been proposed, including abnormal lumbricals; short flexor digitorum superficialis (FDS), which is often accompanied by subsequent or associated skin shortening; tight fascial bands; a deficient dorsal central slip ex- tensor mechanism; and changes in the distal interphalangeal joint or metacarpophalangeal joint [3]. Camptodactyly can have an early or late onset, and it has been proven to show an autosomal dominant pattern of inheritance [4]. This condition often does not cause functional impairment, meaning that patients seek medical attention for concerns relating to cosmetic appearance or military recruitment. Nonoperative and operative techniques have been proposed to treat this condition, depending on its clinical severity. These diverse techniques range from splinting or stretching exercises to release of tendons, fascial bands, transfer of muscles, and tenotomy $[5,6]$. In this arti- 
cle, we present the outcomes of the surgical management of 14 patients with camptodactyly.

\section{METHODS}

The treatment protocol for this condition still lacks standardization. Hence, the aim of this study was to analyze the exact anatomical aetiology and the corresponding plan of the surgical procedure. We also critically evaluated the outcomes depending upon the procedure and reviewed the pre-established protocols. The patients who underwent surgery also underwent normal admission procedures.

The study was carried out on 14 patients with simple camptodactyly without any other deformities, such as clinodactyly. Proper ethical clearance was obtained from the institute (ref. no. AIIMS/Pat/IEC/2016/235). Written informed consent for surgery was duly provided by the patients or by the parents, if the patients were children.

Surgical planning was done for all 14 patients and 15 fingers with a progressive deformity of more than $60^{\circ}$. The preoperative assessment was based on the shortening of skin, the extent of the deformity, and whether the deformity was progressive or static.

Surgery was performed under regional block. The site of the incision was marked. Cases were classified intraoperatively as those having involvement of skin, FDS, lumbricals, fascia, or no anomalies. The intraoperative findings were as follows: skin shortening in two cases, tight and small FDS in 10 cases, tight FDS in two cases, abnormal lumbrical insertion in one case, small FDS and tight fascial bands in one case, and no anomalies

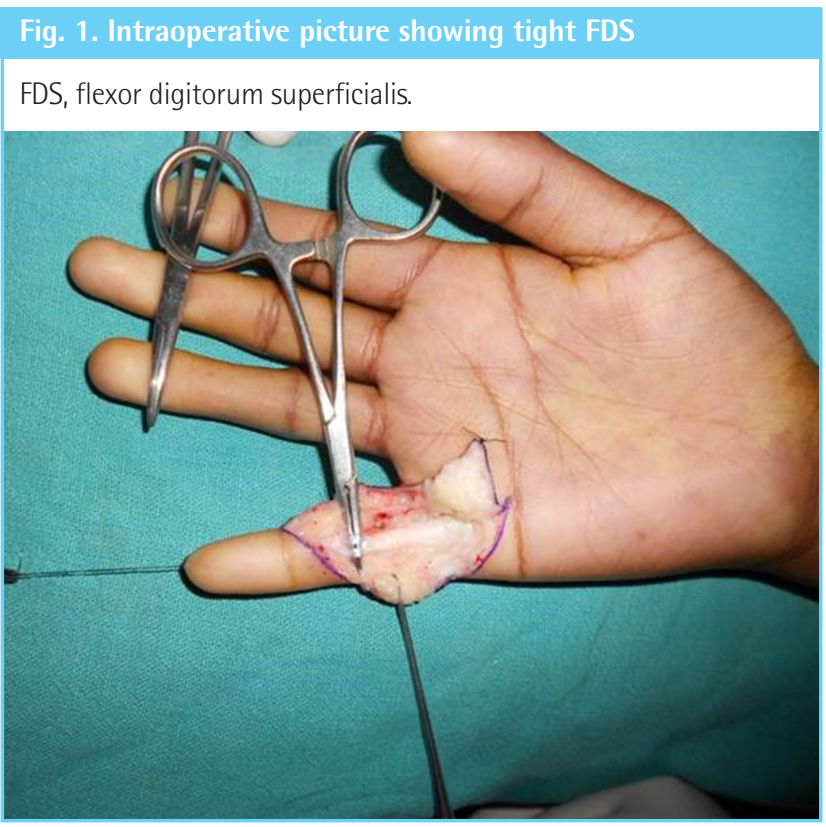

in one case. Thus, the procedures performed were as follows: FDS release alone in seven cases (Figs. 1 and 2), FDS and fascial band release in one case; FDS release and split-thickness skin grafting (SSG)/Z-plasty in two cases, FDS release and transfer to the extensors in three cases, and transfer of anomalous lumbrical insertion to the lateral band in one case (Table 1). The grading system (Mayo Clinic) proposed by Siegert et al. [7] was used to classify the surgical outcomes.

Postoperatively, we decided upon splinting of the finger for 2 weeks, followed by physiotherapy. Patients were followed up at weekly intervals for at least 6 months.

\section{RESULTS}

A total of 14 patients and 15 fingers were operated on. Nine patients were females (64.29\%) and five were males (35.71\%).

Eleven patients had unilateral camptodactyly (78.57\%), while three were affected bilaterally; one amongst the diseased had bilateral camptodactyly of four fingers (ring and little fingers of both hands). A positive family history could be traced in three of the patients $(21.43 \%)$. Of the 14 patients, 13 (92.86\%) were right-handed and one (7.14\%) had left dominance. The mean age of the surgical group ranged from 7 to 23 years, with an average of 15 years. The affected little finger was operated on in all patients, with a preoperative mean extension lag of $50^{\circ}$ (range, $30^{\circ}-90^{\circ}$ ) (Fig. 3). The mean follow-up duration was 15 months (range, 6-28 months).

Three of the 14 patients (21.43\%) developed ankylosis, and four $(28.57 \%)$ showed no changes in flexion even after surgery. Of the seven patients (50\%) who reported improved flexion,

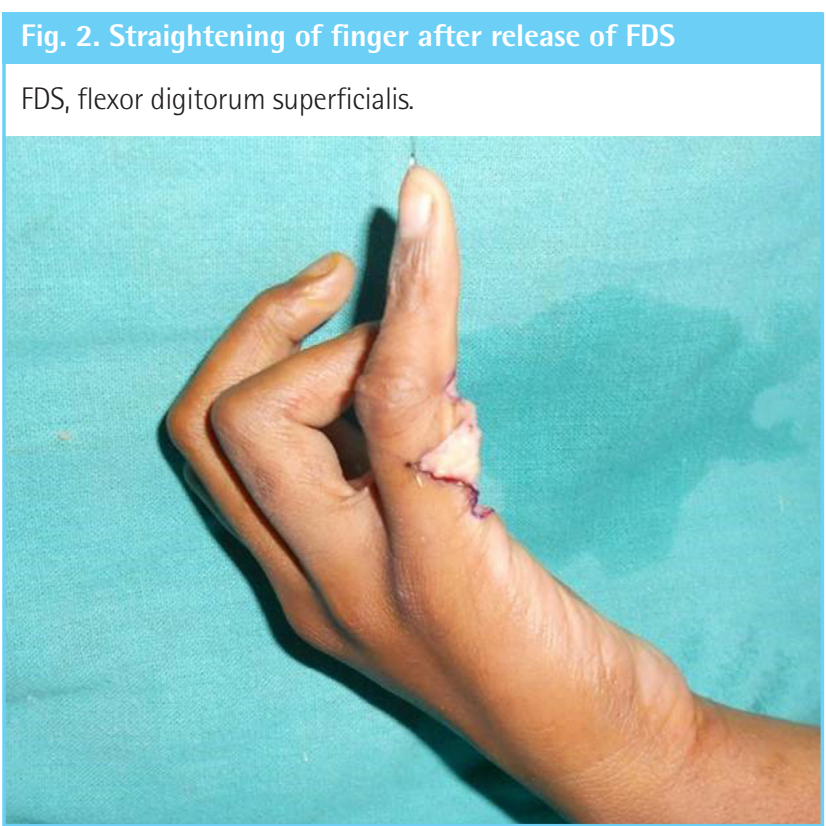


Table 1. Descriptive data of patients

\begin{tabular}{|c|c|c|c|c|c|c|c|c|c|c|}
\hline $\begin{array}{r}\text { Case } \\
\text { No. }\end{array}$ & $\begin{array}{l}\text { Family } \\
\text { history }\end{array}$ & Dominance & $\begin{array}{l}\text { Age at } \\
\text { surgery } \\
\text { (yr) }\end{array}$ & $\begin{array}{l}\text { Treated } \\
\text { digit(s) }\end{array}$ & $\begin{array}{c}\text { Preoperative } \\
\text { extension } \\
\operatorname{lag}\left({ }^{\circ}\right)\end{array}$ & $\begin{array}{l}\text { Operative } \\
\text { findings }\end{array}$ & Procedure & $\begin{array}{l}\text { Postoperative } \\
\text { extension lag } \\
\left({ }^{\circ}\right)\end{array}$ & $\begin{array}{l}\text { Postoperative } \\
\text { effect on } \\
\text { flexion }\end{array}$ & Grade \\
\hline 1 & - & Right & 7 & $\mathrm{LF}$ & 30 & Tight and small FDS & FDS release & 60 & Ankylosis & $P$ \\
\hline 2 & - & Right & 13 & LF & 30 & Tight and small FDS & FDS release & 30 & No effect & $P$ \\
\hline 3 & + & Right & 15 & $\mathrm{LF}(\mathrm{b} / \mathrm{l})$ & 60,60 & Tight and small FDS & FDS release & 15,30 & No effect & $G, F$ \\
\hline 4 & - & Right & 22 & LF & 60 & Tight and small FDS & FDS release & 30 & No effect & $\mathrm{F}$ \\
\hline 5 & - & Right & 23 & LF & 60 & Tight and small FDS & FDS release & 30 & Only $30^{\circ}$ motion & $\mathrm{F}$ \\
\hline 6 & - & Right & 12 & LF & 45 & No anomalies & FDS transfer to extensors & 60 & Only $20^{\circ}$ motion & $P$ \\
\hline 7 & + & Right & 9 & $\mathrm{LF}$ & 30 & $\begin{array}{l}\text { Abnormal lumbrical } \\
\text { insertion }\end{array}$ & Transfer to lateral bands & 20 & Only $10^{\circ}$ motion & $P$ \\
\hline 8 & - & Right & 12 & LF & 30 & Tight and small FDS & Release & 30 & $20^{\circ}$ flexion & $P$ \\
\hline 9 & + & Right & 22 & LF & 90 & $\begin{array}{l}\text { Tight FDS and fascial } \\
\text { bands }\end{array}$ & Release and skin grafting & 30 & $40^{\circ}$ flexion & $\mathrm{F}$ \\
\hline 10 & - & Right & 14 & LF & 45 & Tight and small FDS & FDS Release & 30 & $15^{\circ}$ flexion & $\mathrm{F}$ \\
\hline 11 & - & Left & 15 & $\mathrm{LF}$ & 60 & Tight FDS & $\begin{array}{l}\text { Release+FDS transfer to } \\
\text { extensors }\end{array}$ & 45 & Ankylosis & $\mathrm{P}$ \\
\hline 12 & - & Right & 20 & LF & 60 & Tight and small FDS & Release+skin Z-plasty & 30 & No effect & $\mathrm{F}$ \\
\hline 13 & - & Right & 15 & LF & 30 & Tight and small FDS & FDS release & 30 & $30^{\circ}$ flexion & $P$ \\
\hline 14 & - & Right & 12 & LF & 60 & Tight FDS & $\begin{array}{l}\text { Release and FDS transfer } \\
\text { to extensors }\end{array}$ & 60 & Ankylosis & $P$ \\
\hline
\end{tabular}

\section{Fig. 3. Appearance after release of FDS}

Postoperative appearance of left little finger (after release of flexor digitorum superficialis [FDS]) in a patient with bilateral involvement.

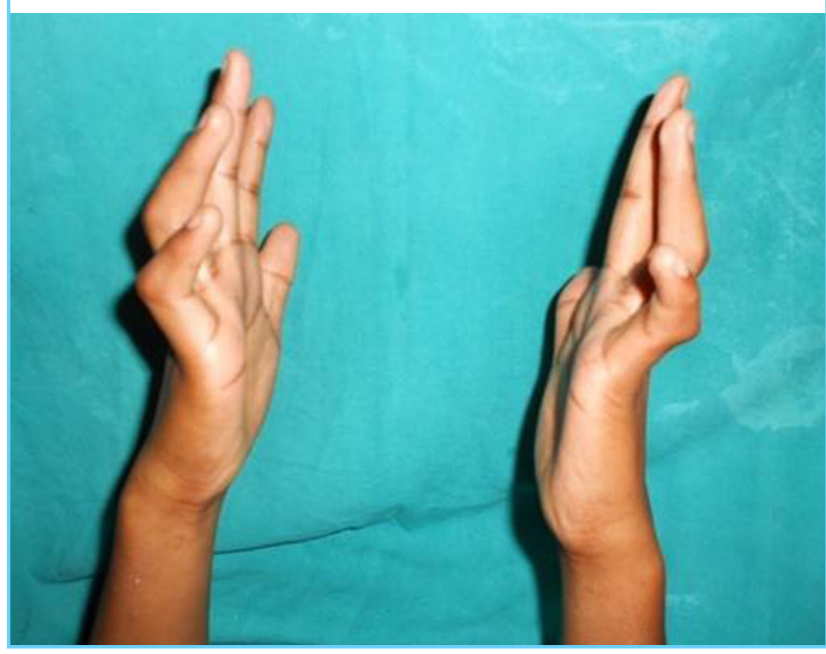

three $(42.86 \%)$ exhibited a definite gain in motion after surgery (Table 1).

\section{DISCUSSION}

Camptodactyly refers to progressive flexion contracture of the PIP joint. It can be simple or complex, if it is associated with other distinct deformities. Benson et al. [8] stated that while patients who presented early with this condition have an equal sex distribution, late-onset patients are mostly females. Additionally, syndromic associations of this condition have also been noted. The little finger is the first to be affected. Other causes of PIP joint flexion, such as Boutonniere deformity, Dupuytren contracture, trigger finger, and an absent extensor mechanism, must be ruled out before confirming the diagnosis. Clinical and radiological parameters are used to define the extent of the deformity and joint flexibility [9]. Based on those factors, surgeons choose between conservative or operative management.

In our study, operative management was planned for all cases with $>60^{\circ}$ of involvement. Further, the type of surgery was decided on the basis of the anatomical defects that were encountered on the operating table. The most commonly affected structure was the FDS, which was short and tight in 10 cases (64.29\%), followed by skin shortening in two cases (14.29\%) and tight FDS alone in two cases (14.29\%).

Of the patients, seven (50\%) underwent FDS release alone, three (21.43\%) underwent FDS release and transfer to the extensors, two (14.29\%) underwent FDS release and SSG cover/ Z-plasty, one (7.14\%) had FDS release along with release of fascial bands, and one (7.14\%) underwent transfer of an anomalous lumbrical insertion to the lateral band. Static splinting was administered for 2 to 3 weeks in cases of tendon transfer, after which patients were taught to engage in gradual mobilisation of the PIP joint for 6 weeks. Night splinting was continued for a prolonged time in all cases.

On the scale (Mayo Clinic) of Siegert et al. [7], we observed that irrespective of the anatomical structure affected and the surgical option used, postoperative functional integrity re- 
mained the same. However, full correction of flexion without ankylosis was not achieved in any patients. Given the growing number of cases of camptodactyly, we chose to review our surgical outcomes. In a comparison with peer groups worldwide, we found that although several procedures have been developed, simple release of the FDS, fascia, or skin mostly suffices, with no need to disturb the extensor mechanism. This, in turn, results in postoperative compromise in flexion.

\section{NOTES}

\section{Conflict of interest}

No potential conflict of interest relevant to this article was reported.

\section{Ethical approval}

The study was approved by the Institute of All India Institute of Medical Sciences Patna (reference no. AIIMS/Pat/IEC/2016/ 235) and performed in accordance with the principles of the Declaration of Helsinki. Written informed consent for surgery was duly provided by the patients or by the parents, if the patients were children.

\section{Patient consent}

The patients provided written informed consent for the publication and the use of their images.

\section{REFERENCES}

1. Choi BR, Lim YH, Joo KB, et al. Camptodactyly, arthropathy, coxa vara, pericarditis (CACP) syndrome: a case report. J Korean Med Sci 2004;19:907-10.

2. Hamilton KL, Netscher DT. Multidigit camptodactyly of the hands and feet: a case study. Hand (N Y) 2013;8:324-9.

3. Santosh R, Haobijam N, Barad AK, et al. Absent flexor digitorum profundus (FDP): an unreported component of camptodactyly. J Med Soc 2014;28:120-2.

4. McFarlane RM, Classen DA, Porte AM, et al. The anatomy and treatment of camptodactyly of the small finger. J Hand Surg Am 1992;17:35-44.

5. Miura T, Nakamura R, Tamura Y. Long-standing extended dynamic splintage and release of an abnormal restraining structure in camptodactyly. J Hand Surg, 1992;17:665-72.

6. Glicenstein J, Haddad R, Guero S. Surgical treatment of camptodactyly. Ann Chir Main Memb Super 1995;14:26471.

7. Siegert JJ, Cooney WP, Dobyns JH. Management of simple camptodactyly.J Hand Surg 1990;15:181-9.

8. Benson LS, Waters PM, Kamil NI, et al. Camptodactyly: classification and results of nonoperative treatment. J Pediatr Orthop 1994;14:814-9.

9. Foucher G, Lorea P, Khouri RK, et al. Camptodactyly as a spectrum of congenital deficiencies: a treatment algorithm based on clinical examination. Plast Reconstr Surg 2006; 117:1897-905. 